\title{
Measurement and determinants of the natural history of liver fibrosis in hepatitis $C$ virus infection: a cross sectional and longitudinal study
}

\author{
M Wright, R Goldin, A Fabre, J Lloyd, H Thomas, C Trepo, P Pradat, M Thursz, on \\ behalf of the HENCORE collaboration
}

See end of article for authors' affiliations

Correspondence to: Dr M Wright, Department of Medicine, Imperial College of Science,

Technology, and Medicine St Mary's Hospital (QEQM Wing), South Wharf Road, London W2 1NY, UK mark.wright@ic.ac.uk

Accepted for publication 29 October 2002
Introduction: The rate of development of liver fibrosis in hepatitis C virus (HCV) infection varies between individuals. This accounts for the variation in duration of progression to cirrhosis. The aims of this study were: (1) to determine whether fibrosis progresses linearly through the grading scales and (2) to identify factors which influence the rate of fibrosis.

Methods: HCV infected patients who had undergone at least one liver biopsy were identified. Biopsies were scored using the modified HAI (Ishak) and METAVIR systems, which were compared. Patients were treatment naïve at first biopsy. Demographic features were examined for their relationship to fibrosis rate (defined as fibrosis stage/infection duration) using univariate and multivariate analysis. A subgroup of patients with two biopsies was examined to test the assumption that fibrosis progresses in a linear fashion.

Results: A total of 917 patients were included. Male sex $(p<0.00001)$, older age at infection $(p \leqslant 0.00001)$, and viral genotype non- $1(p=0.005)$ were all associated with a rapid rate of fibrosis. On multiple linear regression they accounted for $29.5 \%$ of the variability in fibrosis rate $\left(r^{2}=0.295\right)$. METAVIR and Ishak scores were highly correlated ( $r=0.935, p<0.0001)$. In 137 patients who had two biopsies, the predicted probability for an increase of 1 on the fibrosis score was too low to assess linearity.

Conclusions: Demographic features account for a minority of fibrosis rate variability. The Ishak and METAVIR scoring systems are equivalent. Linearity of fibrosis progression cannot be assessed in biopsies only a few years apart.
$\mathrm{H}$ epatitis C virus (HCV) is a hepatotrophic flavivirus infecting 170 million people worldwide. It is a major cause of cirrhosis and hepatocellular carcinoma, and is now a leading indication for liver transplantation. The rate of progression to cirrhosis varies among individuals, and the natural history of HCV has been a subject of controversy, with conflicting data based on cohort and clinic based studies. Some authors have suggested that the majority of those infected will suffer no long term sequelae ${ }^{12}$ while others have suggested a more rapid course. ${ }^{3}$ Poynard and colleagues ${ }^{4}$ published a large scale study in which they defined three groups of patients with slow, moderate, or rapid fibrosis. In addition, major risk factors were identified: male sex, excess alcohol, and age greater than 40 years at acquisition.

At initial diagnosis it is not possible to classify patients into these groups and therefore determine who to treat urgently and who to treat at all. The treatment of those infected with HCV is currently focused on attempting to eradicate the virus and there is a balance to be struck between treating all (thereby giving side effects to a large number of patients many of whom will not benefit) and attempting to target therapy to patients at high risk. Currently, a histology based strategy is in widespread use, treatment being determined by degree of fibrosis and inflammation on presenting liver biopsy and the amount of change between subsequent biopsies. ${ }^{5-7}$ This involves taking serial liver biopsies several years apart. Optimal timing is not established and there are a number of problems with this approach, given our current limited understanding of fibrosis progression: there may be a sampling error ${ }^{89}$ and interpretation is subject to interobserver variation.
The rate of fibrosis can be calculated, based on fibrosis score at biopsy, if the date of infection is known and we assume fibrosis score is zero at the time of infection:

fibrosis score at biopsy/years infected at the time of biopsy.

This represents the average rate of fibrosis in an individual up to the time of biopsy. There are probably periods of slower and faster fibrosis throughout the period of infection and possibly even periods of regression. At present, we can only calculate this rate retrospectively and any assumption regarding the future rate of fibrosis is conjecture. As a set of working hypotheses we can predict at least three possible patterns of fibrosis progression. If fibrosis progression is linear throughout infection, there will be different rates for different individuals, but if date of infection and degree of fibrosis is known, then it is simple to predict prognosis. If the bulk of fibrosis occurs early it is possible to divide patients into groups of mild, moderate, and severe, as we do now, and prognosticate on this basis provided that sufficient time has elapsed since infection. Finally, the fibrotic process may suddenly take off after a variable period of time. If this is the case then liver biopsy may be of little value in making future predictions. This subject is therefore worthy of further study.

Fibrosis scores are derived by histopathologists using semiquantitative scoring systems (see Desmet and colleagues ${ }^{10}$ and Brunt and colleagues ${ }^{11}$ for review). The METAVIR ${ }^{12}$ and 
modified HAI (Ishak) $)^{13}$ systems are the most widely used and assign numeric (integer) values to stage fibrosis based on architectural changes and to grade inflammation based on the degree of necroinflammatory lesions. These systems use ordinal variables (a fibrosis score of 4 does not equal twice as much collagen as a score of 2 for example) and cannot be assumed to represent quantitative data. To use morphological features to calculate rate seems intrinsically artificial. In the absence of an alternative, Poynard and colleagues ${ }^{4}$ described linear progression of fibrosis using the METAVIR system. However, later work by this group has suggested an acceleration of the rate later in life. ${ }^{14}$ Most of this work relies on only two points of data (that is, point of infection and first biopsy), and attempts to extrapolate longitudinal results from cross sectional data. Different semiquantitative scoring systems may show different patterns of progression according to how those systems are constructed. In order to more completely describe the natural history of fibrosis it is necessary to examine relationships of fibrosis against time using three or more data points.

We sought to expand the knowledge in this area. Using a large database we have analysed the relationships between rate of fibrosis deposition and demographic features. We demonstrate the impact that different scoring systems may have on the way fibrosis appears to progress. We have explored the utility of a second biopsy for demonstrating how fibrosis progresses in an individual and the optimal timing between biopsies.

\section{METHODS}

\section{Patients}

All patients with HCV infection seen at St Mary's Hospital who had undergone at least one liver biopsy between 1 January 1990 and 30 June 2001 were identified. A subset of patients who had undergone multiple biopsies was identified and listed. All patients were known to be HCV antibody and RNA positive. Exclusion criteria were hepatocellular carcinoma, other types of liver disease in addition to their hepatitis $\mathrm{C}$, human immunodeficiency virus infection, treatment prior to first biopsy, and non-interpretable biopsy.

Data were collected with regard to patient demographics (sex, date of birth, age at infection, current alcohol intake, risk factors, ethnic origin), histological data (dates and scores), virological features (HCV genotype), and antiviral therapy (dates and types). Where possible, date of infection was documented. In those infected by blood products the exact date was recorded where known. When the exact date was not known, but the year was, the estimated date of infection was recorded as the middle of that year. In patients infected by intravenous drug use, the date of infection was estimated as the middle of the year of first drug use, as elsewhere. ${ }^{4}$ Locally derived data were combined with those of a pan European collection from the HENCORE ${ }^{15}$ study to produce a master database.

\section{Histopathology}

Sections were stained with haematoxylin and eosin and a reticulin stain. For the subset of patients with two or more biopsies, each biopsy was scored using both METAVIR and the modified HAI (Ishak) systems by two histopathologists working together at the time of biopsy review and reaching agreement. The METAVIR system uses a five point scale for fibrosis stage $(0-4)$ and a four point scale for activity $(0-3)$. The modified HAI system (Ishak) uses a seven point scale for fibrosis stage (0-6) and an 18 point scale for activity. The scores by each system were then analysed to check for correlation.

Intra and interobserver reproducibility for fibrosis estimation was examined using weighted kappa analysis after repeated scoring of 30 of the 274 slides scored.

\section{Plan of analysis}

All statistics were performed using SPSSv10 (SPSS Inc., Chicago Illinois, USA). Biopsy data and demographic details were summarised for the cohort as a whole (table 1) and
Table 1 Demographic features of patients with at least one liver biopsy

\begin{tabular}{|c|c|c|}
\hline Characteristic & Total & \\
\hline No of patients (\%) & 1606 & \\
\hline Males & 940 & (58.5) \\
\hline Females & 666 & (41.5) \\
\hline \multicolumn{3}{|c|}{ Age at infection ( $n=917)$} \\
\hline Mean (SD) & 27.7 & (11.8) \\
\hline Median & 24 & (0-72) \\
\hline \multicolumn{3}{|c|}{ Necroinflammatory score (lshak) } \\
\hline Mean (SD) & \multicolumn{2}{|c|}{$4.48(2.1)$} \\
\hline Median & 4 & $(0-11)$ \\
\hline \multicolumn{3}{|l|}{ Fibrosis score (lshak) } \\
\hline Mean (SD) & \multicolumn{2}{|c|}{$2.72(1.7)$} \\
\hline Median & 2 & $(0-6)$ \\
\hline \multicolumn{3}{|c|}{ Duration infection to 1 st biopsy } \\
\hline Mean (SD) & 15.1 & $(9.9)$ \\
\hline Median & 14 & $(0-57)$ \\
\hline \multicolumn{3}{|c|}{ Rate (fibrosis units/year) } \\
\hline Mean (SD) & \multicolumn{2}{|c|}{$0.39(1.28)$} \\
\hline Median & \multicolumn{2}{|c|}{$0.17(0-6)$} \\
\hline \multicolumn{3}{|l|}{ Risk factors (\%) } \\
\hline Unknown & 622 & (43.8) \\
\hline Blood & 433 & $(27.8)$ \\
\hline IVDU & 430 & $(26.8)$ \\
\hline Multiple & 25 & $(1.6)$ \\
\hline \multicolumn{3}{|c|}{ Alcohol consumption (units/week) (\%) } \\
\hline Unknown & 377 & $(23.5)$ \\
\hline Nil & 799 & (49.8) \\
\hline$<20$ & 256 & (15.9) \\
\hline $20-40$ & 62 & $(3.9)$ \\
\hline $40+$ & 112 & (9.1) \\
\hline \multicolumn{3}{|l|}{ Ethnic origin (\%) } \\
\hline Caucasian & 1400 & (87.2) \\
\hline Non-Caucasian & 206 & (12.8) \\
\hline \multicolumn{3}{|l|}{ Viral genotype (\%) } \\
\hline 1 & 652 & $(40.6)$ \\
\hline Non-1 & 330 & $(20.5)$ \\
\hline Unknown & 624 & (38.9) \\
\hline
\end{tabular}

tested for normality. The distribution of the rates of fibrosis was skewed and therefore was logarithmically transformed in order to normalise the data. Fibrosis rate is defined as: the ratio of the change in fibrosis stage to the duration of infection between assessments. Fibrosis stage is assumed to be zero at the point of infection.

In the dataset as a whole (see tables 2 and 3 ) factors related to the severity and rate of fibrosis were analysed. Effect of viral genotype, mode of transmission, alcohol intake, racial group, and sex on fibrosis score were analysed using the $\chi^{2}$ test. For patients in whom date of infection was known, their effect on the fibrosis rate was analysed using the Mann-Whitney U test. A logarithmic transformation of the rate was performed to achieve a normal distribution. The effect of age at infection and at biopsy on the stage and rate of fibrosis were analysed using analysis of variance after logarithmic transformation of the rate. Patient age was stratified by decade of life at infection and biopsy.

Those factors significantly associated with the rate of fibrosis on univariate analysis were incorporated into a stepwise multiple regression model.

For patients who had undergone more than one biopsy, rates of fibrosis were calculated for the period between infection and the first biopsy (R1) and between the first and second biopsies (R2). Patients were stratified according to whether they had been treated between biopsies or not.

\section{RESULTS}

Effect of the scoring system used

A total of 274 biopsies were assessed using both the METAVIR and Ishak semiquantitative scores. The correlation between 
Table 2 Demographic features and progression of fibrosis

\begin{tabular}{|c|c|c|c|c|c|c|}
\hline & \multicolumn{2}{|c|}{ Fibrosis score } & \multirow[b]{2}{*}{$\mathrm{p}$ Value } & \multicolumn{2}{|c|}{ Rate of fibrosis } & \multirow[b]{2}{*}{$p$ Value } \\
\hline & $\mathrm{n}$ & Fibrosis score* & & $\mathrm{n}$ & Fibrosis score & \\
\hline \multicolumn{7}{|l|}{ Sex } \\
\hline Male & 940 & $2.88(1.74)$ & & 511 & $0.40(0.73)$ & \\
\hline Female & 666 & $2.49(1.62)$ & $<0.00001+$ & 387 & $0.29(0.58)$ & $<0.00001 \neq$ \\
\hline \multicolumn{7}{|l|}{ Alcohol (per week) } \\
\hline Nil & 799 & $2.69(1.60)$ & & 429 & $0.36(0.69)$ & \\
\hline$<20$ units & 256 & $2.63(1.67)$ & & 172 & $0.42(0.72)$ & \\
\hline $20-40$ units & 62 & $2.65(1.78)$ & & 41 & $0.38(0.76)$ & \\
\hline $40+$ units & 112 & $3.12(1.96)$ & $0.02 ף$ & 83 & $0.29(0.46)$ & $0.829 \ddagger$ \\
\hline \multicolumn{7}{|l|}{ Race } \\
\hline Caucasian & 1400 & $2.71(1.68)$ & & 818 & $0.36(0.69)$ & \\
\hline Non-Caucasian & 206 & $2.74(1.82)$ & $0.823 t$ & 81 & $0.22(0.31)$ & $0.07 \ddagger$ \\
\hline \multicolumn{7}{|l|}{ Viral genotype } \\
\hline 1 & 652 & $2.8 \quad(1.66)$ & & 347 & $0.31(0.5)$ & \\
\hline Non-1 & 330 & $2.85(1.56)$ & $0.704 \dagger$ & 194 & $0.47(0.82)$ & $0.005 \ddagger$ \\
\hline \multicolumn{7}{|c|}{$\begin{array}{l}\text { *Mean (SD). } \\
\text { †Independant sample } t \text { test. } \\
\neq \text { Mann-Whitney U test. } \\
\text { ॠAnalysis of variance (linear trend). }\end{array}$} \\
\hline
\end{tabular}

\begin{tabular}{|c|c|c|}
\hline & Fibrosis score & Rate of fibrosis \\
\hline $\begin{array}{l}\text { Age at infection }(y)(n=917) \\
27.7(11.8) \text { (mean (SD)) }\end{array}$ & $p<0.000019$ & $p<0.000019$ \\
\hline $\begin{array}{c}\text { Age at biopsy }(y)(n=1606) \\
42.2(11.6)(\text { mean }(S D))\end{array}$ & $p<0.000019$ & $p=0.312 \pi$ \\
\hline
\end{tabular}

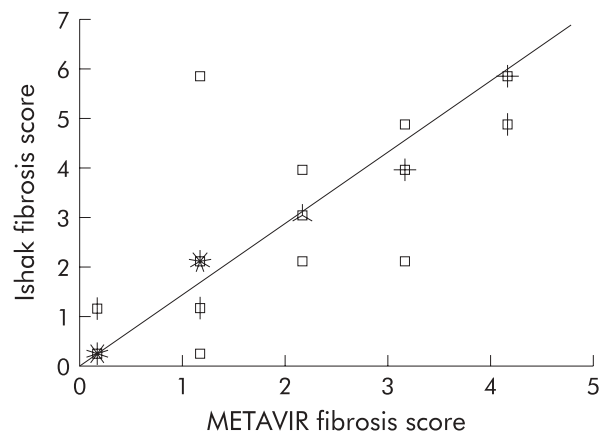

Figure 1 Scatter plot of the modified HAl score against the METAVIR score. A total of 274 biopsies were assessed: $r=0.959$, $p<0.00001$. (Each spike on a point represents five cases.)

them was high ( $r=0.96 \mathrm{p}<0.00001)$ (fig 1$)$. This suggests that apparent differences in the natural history of HCV observed between groups using these scoring systems is unlikely to be due to the scoring systems themselves.

\section{Reproducibility of semiquantitative scoring system}

There was good intra and interobserver agreement (weighted kappa 0.87 and 0.82 , respectively) on the 274 biopsies.

\section{Results for the dataset as a whole}

A total of 1606 patients had data available for analysis. Demographic features are shown in table 1. Age at infection, age at biopsy, duration of infection at biopsy, and fibrosis score were all found to have approximately normal distributions. The rate of fibrosis had a non- normal distribution with a marked right skew. Logarithmic transformation was therefore carried out as required prior to analyses. Based on the calculated rate of fibrosis, the mean time to cirrhosis was 15 years and the

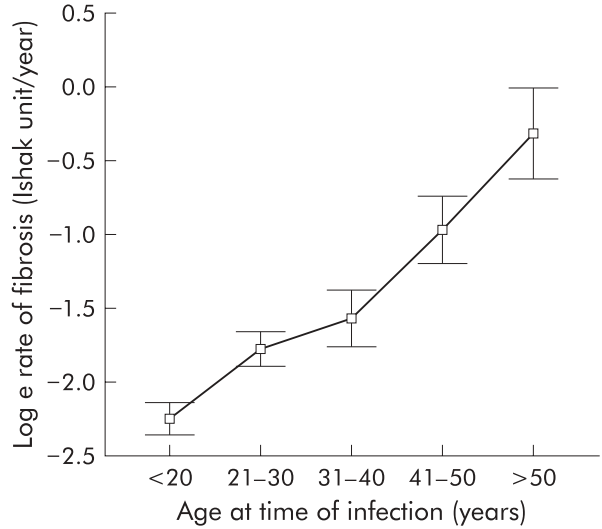

Figure 2 Age at time of infection plotted against the log of fibrosis rate (error bars represent $95 \%$ confidence interval).

median was 35 years (in close agreement with other work ${ }^{4}$ ). Male sex $(\mathrm{p}<0.00001)$ and older age at infection $(\mathrm{p}<0.00001)$ were associated with both increased fibrosis severity and a faster rate of fibrosis. Heavy alcohol intake $(p=0.02)$ and older age at biopsy $(\mathrm{p}<0.00001)$ were associated with increased fibrosis severity. Viral genotype non- 1 was associated with a faster rate but not overall severity (table 2 ). Increasing fibrosis score with age at biopsy and duration of infection both demonstrated the progressive nature of fibrosis in HCV infection. There was a linear relationship between age at infection and rate of fibrosis (fig 2) but no such relationship between age at biopsy and rate of fibrosis (fig 3), implying that an individual's rate of fibrosis does not vary with their age.

When duration of infection was plotted against fibrosis score, steady progression was again demonstrated (fig 4).

Fibrosis score was found to increase in step with mean age at biopsy and demonstrated a linear trend $(\mathrm{p}<0.00001$, ANOVA) (fig 5). Mean rate of fibrosis calculated by dividing the change in fibrosis score by the difference in mean ages with those scores (that is (score 2 -score 1 )/(mean age at score 2 -mean age at score 1)) gave a rate of 0.43 fibrosis units/year ( 14 years to cirrhosis) which was similar to that for the cohort as a whole.

\section{Construction of a regression model to predict the rate of fibrosis}

Factors on univariate analysis related to the rate of fibrosis (age at infection, sex, and viral genotype (table 2)) were entered by the forward stepwise method into a multiple 


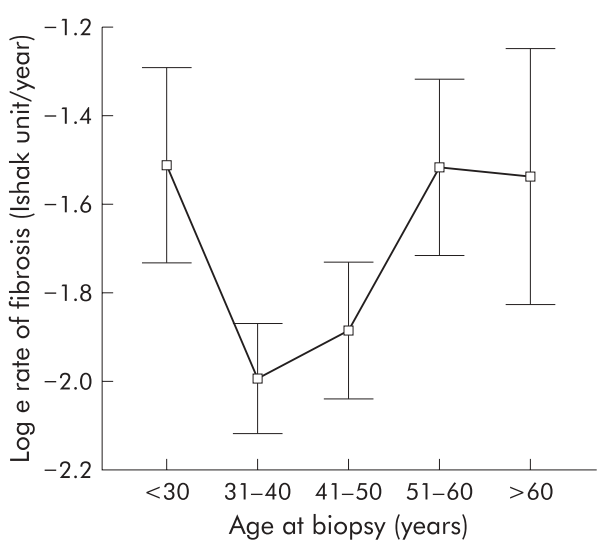

Figure 3 Age at time of biopsy plotted against the log of fibrosis rate (error bars represent $95 \%$ confidence interval).

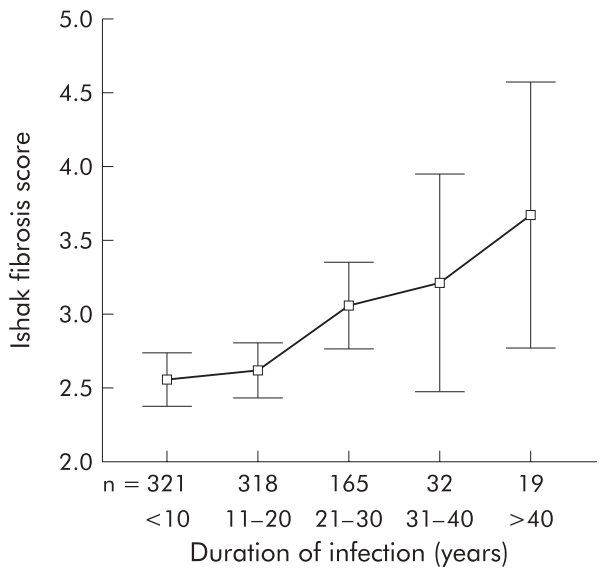

Figure 4 Duration of infection plotted against fibrosis score at the first biopsy.

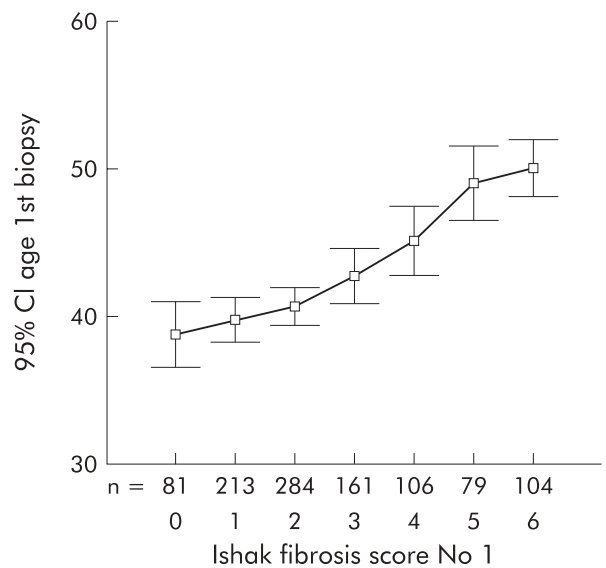

Figure 5 Mean age at each fibrosis stage.

regression model to attempt prediction of $\log _{\mathrm{e}}$ fibrosis rate for individuals (table 4 ). This model accounted for only $29.5 \%$ of the variability in fibrosis rate $\left(r^{2}=0.295\right)$.

\section{Patients with two or more biopsies}

A total of 137 patients had undergone two or more biopsies: 93 of these patients were untreated and 44 had received therapy (interferon monotherapy or interferon ribavirin combination therapy) between the first and second biopsy. There was a considerable difference between the time to the first biopsy
Table 4 Linear regression model to analyse predictors for rate of fibrosis $(517$ patients had data for all three factors)

\begin{tabular}{|c|c|c|c|}
\hline $\begin{array}{l}\text { Factors included in the } \\
\text { model }\end{array}$ & $\begin{array}{l}\text { Unstandardised } \\
\text { coefficient }\end{array}$ & $\mathrm{p}$ Value & $\begin{array}{l}r^{2} \text { for the } \\
\text { model }\end{array}$ \\
\hline $\begin{array}{l}\text { Age at infection } \\
\text { Sex } \\
\text { Viral genotype }\end{array}$ & $\begin{array}{l}0.043 \text { (SE 0.003) } \\
0.326 \text { (SE 0.66) } \\
0.251 \text { (SE 0.88) }\end{array}$ & $\begin{array}{l}<0.00001 \\
0.0002 \\
0.005\end{array}$ & 0295 \\
\hline
\end{tabular}

and duration between biopsies (15.6 $v 2.73$ years for the untreated group and $16.9 \vee 2.69$ years for the treated group).

Rates of fibrosis between infection and the first biopsy and between the first and second biopsy were calculated. Patients treated between the first and second biopsy were analysed separately from those who remained untreated. The treatment group had significantly worse liver disease than the untreated group, with higher mean fibrosis stage on their first biopsy $(p=0.005$ by the Ishak system) (see table 5$)$. Because of this difference, observations of the effect of therapy on the rate of fibrosis compared with no therapy should be interpreted with caution. Likewise, natural history data from the untreated group must be treated with caution as these patients, on average, had mild disease and may not reflect all patients with HCV.

There were no demographic differences between the untreated and treated group (see table 5).

If the rate of fibrosis is constant with time, then the rate between infection and the first biopsy (Rl) will be the same as between the first and second biopsy (R2). If $\mathrm{R} 2$ is greater than $\mathrm{Rl}$, this suggests that the rate of fibrosis increases with time. If $\mathrm{R} 1$ is greater than $\mathrm{R} 2$, this suggests that the rate of fibrosis decreases with time.

For scoring systems using discrete variables there is a probability of an individual moving from one category to another in a given time. HCV is a slowly progressive disease. If we look at the average fibrosis rates-for example, in untreated patients scored using the modified HAI, 0.15 units/year-we would not expect the fibrosis score to increase by 1 unit for about seven years, and so for a mean time between biopsies of only 2.7 years the mean probability of a change in score was low. This was even more pronounced for METAVIR where there are fewer stages. As a result of the apparent non-progression between biopsies, the average rate of fibrosis falls artefactually, as observed in our population (table 6).

To avoid this problem, the expected fibrosis score of the second biopsy (rounded up or down to the nearest whole number) was calculated from Rl and compared with the observed score. In this way if the time between biopsies was short, we expected to see no change in score. If the observed score was higher or lower than the calculated expected value, we inferred acceleration or deceleration of the fibrosis rate. Observed and expected scores were then compared using a paired sample $t$ test. The null hypothesis was: that there will be no difference between observed and expected (based on a constant rate of infection) fibrosis scores for the second biopsy. Comparison of observed scores on the second biopsy with those expected on the basis of a constant fibrosis rate are shown in table 7 . The null hypothesis that there will be no difference between observed and expected scores is accepted for the untreated group (NS). For the treated group there was a significant departure (decrease) from a linear rate demonstrating an effect of therapy. For the METAVIR score, significance was not reached, independent of treatment outcome. 
Table 5 Demographic features of patients with two or more liver biopsies

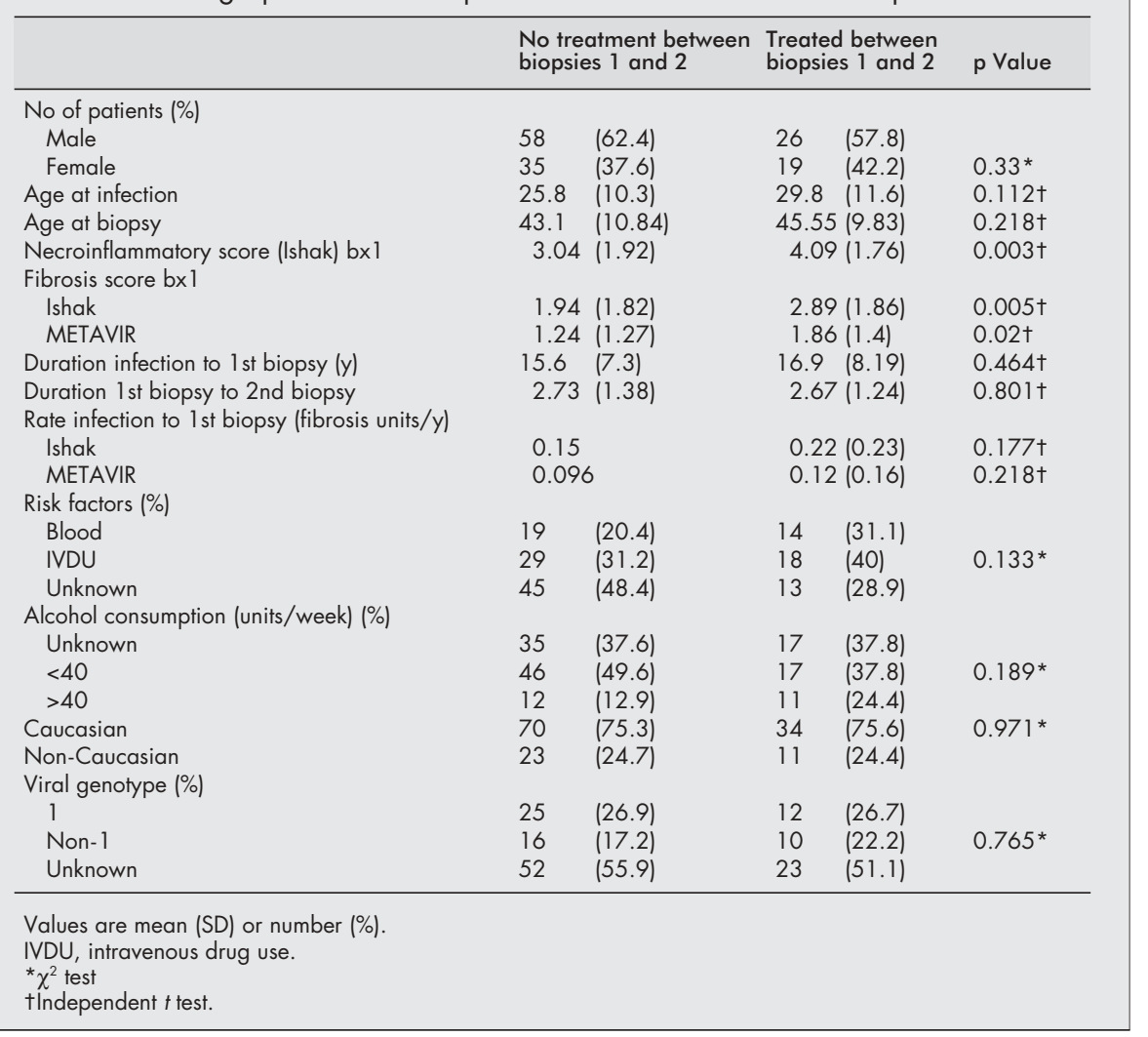

Table 6 Apparent fibrosis rate falls between biopsy 1 and 2 as a function of the short duration between these times

\begin{tabular}{lllll}
\hline Ishak & $\begin{array}{l}\text { Rate 0-1 (R1) } \\
\text { (units/y) }\end{array}$ & $\begin{array}{l}\text { Fibrosis biopsy 1 } \\
\text { (units/y) }\end{array}$ & $\begin{array}{l}\text { Rate 1-2 (R2) } \\
\text { (units/y) }\end{array}$ & $\begin{array}{l}\text { Fibrosis biopsy 2 } \\
\text { (units/y) }\end{array}$ \\
\hline $\begin{array}{l}\text { Untreated }(\mathrm{n}=93) \\
\quad \text { Mean }\end{array}$ & 0.147 & 1.94 & 0.022 & 2.02 \\
$\quad$ SD & 0.24 & 1.82 & 0.783 & 1.88 \\
$\quad \begin{array}{l}\text { Range } \\
\text { Treated }(\mathrm{n}=44)\end{array}$ & $0-1.3$ & $0-6$ & $-2.35-3.46$ & $0-6$ \\
$\quad$ Mean & 0.218 & 2.89 & -0.126 & 2.73 \\
SD & 0.22 & 1.86 & 0.78 & 1.86 \\
Range & $0-1.18$ & $0-6$ & $-2.94-1.6$ & $0-6$ \\
\hline & & & \\
\hline
\end{tabular}

Table 7 Comparison of observed fibrosis score at second biopsy compared with that expected based on the rate of fibrosis between the time of infection and the first biopsy

\begin{tabular}{lll}
\hline & $\begin{array}{l}\text { Difference between observed and } \\
\text { expected fibrosis score for biopsy 2 } \\
\text { (means) }\end{array}$ & p Value \\
\hline $\begin{array}{l}\text { Untreated } \\
\text { Treated }\end{array}$ & Ishak (O) 1.77, (E) 1.62 & NS \\
\hline & Ishak (O) 2.61, (E) 3.21 & 0.03 \\
\hline
\end{tabular}

\section{DISCUSSION}

As with other large studies, the fibrosis process is shown to be progressive and faster for males and those who acquire the infection later in life. Alcohol intake does not appear to influence the rate of fibrosis in this patient group, although it is related to stage. Data collection in this area is notoriously inaccurate and approximately half of the cohort reported abstinence. Selective recall by those with more severe disease could give an association with severity without demonstrating an effect on rate. We were only able to record current alcohol intake and patients aware of their diagnosis may have modified their drinking behaviour in accordance with medical advice. Another possible confounder is that all patients in the cohort had data for stage whereas only $56.2 \%$ had data for rate of fibrosis. The association of viral genotype non-1 with rapid fibrosis has not been noted previously in studies of this type. The lack of association between viral genotype and extent of fibrosis may reflect changing patterns in the epidemiology of HCV infection, with stage lagging behind rate. Patients presenting with genotype 1 may have acquired it through intravenous drug use and therefore were younger at infection, which protects them against fast fibrosis.

A key question we have attempted to answer is whether or not the fibrosis rate is linear-that is, constant throughout life. In favour of a linear fibrosis rate are the steady (and linear) increase in fibrosis score with duration of infection at biopsy, the steady increase in mean age for a given fibrosis stage, and the lack of a relationship between age at biopsy and 
rate of fibrosis (meaning that a patient's rate of fibrosis at a given time does not depend on how old they are). Limited results from the dual biopsies showed no difference between the observed fibrosis score of the second biopsy and that estimated from the rate from time of infection to first biopsy.

The main points demonstrated from the serial biopsy data were the methodological problems. Patients who remain untreated between biopsies have less severe liver disease than those who are treated, meaning that we can only look at the natural history in patients with (relatively) mild disease. The short time between the first and second biopsy, and the discrete and bounded nature of the variables (fibrosis score) means that it is unlikely that progression will be observed in many cases where it is (slowly) occurring. It therefore appears that multiple biopsies using semiquantitative scores are too blunt an instrument unless taken many years apart. Although it would be desirable to take a large number of biopsies at wider intervals to study the natural history, this is not in keeping with ethical or clinical reality.

This has clear clinical implications: whereas European guidelines suggest repeat biopsy every 3-5 years, these data suggest a much longer interval is needed if progression is to be observed. One cannot be reassured by the fact that an individual's fibrosis score has not progressed between liver biopsies only a few years apart.

An acknowledged problem of all studies of this type is the reliability of the date of infection and the assumption that fibrosis is zero at the date of infection.

Attempts to obtain truly quantitative measures of fibrosis from livers biopsies using collagen specific stains and digital image analysis have been unsuccessful due to lack of reproducibility. ${ }^{16}$ Serological markers of fibrosis also have limited utility as the coefficients of variation are wide. ${ }^{17}$ We conclude that, for the time being, we must rely on the morphological assessment of fibrosis on which to base estimates of rate of progression. However, biopsies taken only a short period of time apart are unlikely to provide useful information to inform clinical decisions or to progress the study of the natural history of HCV infection.

The regression model based on the data set here accounts for only $29.5 \%$ of the variability in fibrosis rate. Although this represents a significant advance on the $17 \%$ quoted in previous studies, ${ }^{4}$ it is clear that other factors, such as host genetic variability, must play an important role in determining the rate of fibrosis.

\section{ACKNOWLEDGEMENTS}

HENCORE is the Hepatitis C European Network for Cooperative Research. HENCORE has received educational grants from the European Commission DG5 and Schering Plough PLC. The participants are: Juan Esteban, MD, Stephanos Hadziyannis, MD, Michael Manns,
PhD, Hans Tillmann, MD, Alfredo Alberti, MD, Liliana Chemello, MD, Giorgio Saracco, MD, Mario Rizzetto, PhD, Jean-Henrik Braconier, MD.

M Wright is a Medical Research Council of Great Britain clinical training fellow. Part of this work was generously supported by funds donated by the Charmot-Horton Foundation. We are grateful to Dr G Foster, Dr J Main, and Dr S Taylor-Robinson (Hepatologists at St Mary's Hospital, London) for the opportunity to study their patients as well as those of the other authors.

\section{Authors' affiliations}

M Wright, R Goldin, A Fabre, J Lloyd, H Thomas, M Thursz, Hepatology Section, Faculty of Medicine, Imperial College, London W2 INY, UK

C Trepo, P Pradat, Department of Gastroenterology, Hotel-Dieu, 1 Place de I'Hopital, F-69288 Lyon, Cedex 02, France

\section{REFERENCES}

1 Seeff LB, Buskell-Bales Z, Wright EC, et al. Long-term mortality after transfusion- associated non-A, non-B hepatitis. The National Heart, Lung, and Blood Institute Study Group. N Engl J Med 1992;327:1906-11.

2 Seeff LB, Miller RN, Rabkin CS, et al. 45-year follow-up of hepatitis C virus infection in healthy young adults. Ann Intern Med 2000:132:105-11.

3 Tong MJ, el Farra NS, Reikes AR, et al. Clinical outcomes after transfusion- associated hepatitis C. N Engl J Med 1995;332:1463-6.

4 Poynard T, Bedossa P, Opolon P. Natural history of liver fibrosis progression in patients with chronic hepatitis C. The OBSVIRC, METAVIR CLINIVIR, and DOSVIRC groups. Lancet 1997;349:825-32.

5 Foster GR, Goldin RD, Main J, et al. Management of chronic hepatitis C: clinical audit of biopsy based management algorithm. BM $1997 \cdot 315: 453-8$

6 EASL International Consensus Conference on Hepatitis C. Paris, 26-28, February 1999, Consensus Statement. European Association for 26-28, February 1999, Consensus Statement. Eur
the Study of the Liver. J Hepatol 1999;30:956-61.

7 Booth JC, Brown JL, Thomas HC. The management of chronic hepatitis C virus infection. Gut 1995;37:449-54.

8 Garrido MC, Hubscher SG. Accuracy of staging in primary biliary cirrhosis. J Clin Pathol 1996:49:556-9.

9 Imamura H, Kawasaki S, Bandai Y, et al. Comparison between wedge and needle biopsies for evaluating the degree of cirrhosis. J Hepatol $1993 \cdot 17 \cdot 215-19$

10 Desmet VJ, Gerber M, Hoofnagle JH, et al. Classification of chronic hepatitis: diagnosis, grading and staging. Hepatology 1994; 19:1513-20.

11 Brunt EM. Grading and staging the histopathological lesions of chronic hepatitis: the Knodell histology activity index and beyond. Hepatology $2000 \cdot 31: 241-6$

12 Intraobserver and interobserver variations in liver biopsy interpretation in patients with chronic hepatitis C. The French METAVIR Cooperative Study Group. Hepatology 1994;20:15-20.

13 Ishak K, Baptista A, Bianchi L, et al. Histological grading and staging of chronic hepatitis. J Hepatol 1995;22:696-9.

14 Poynard T, Ratziu V, Charlotte F, et al. Rates and risk factors of liver fibrosis progression in patients with chronic hepatitis c. J Hepatol 2001;34:730-9.

15 Hencore Report. European community. Directorat General (V), 1997.

16 Wright M, Thursz MR, Pullen R, et al. Quantitative versus morphological assessment of liver fibrosis: semi-quantitative scores are more robust than digital image fibrosis area estimation. Liver Int 2003;23:28-34.

17 Poynard T, Imbert-Bismut F, Ratziu V, et al. Biochemical markers of liver fibrosis in patients infected by hepatitis $C$ virus: longitudinal validation in a randomized trial. J Viral Hepat 2002;9:128-33. 